\title{
The Effect of Perlite Addition on Erosive Wear Behavior of Alumina Ceramics
}

\author{
S. TAS ${ }^{a, *}$, E. ERCENK ${ }^{b}$ AND S. YILMAZ ${ }^{b}$ \\ ${ }^{a}$ Istanbul Technical University (ITU), Department of Mechanical Engineering, Gumussuyu, Istanbul 34437, Turkey \\ ${ }^{b}$ Sakarya University, Engineering Faculty, Department of Metallurgical and Materials Engineering \\ Esentepe Campus, 54187 Sakarya, Turkey
}

\begin{abstract}
In this study, the effects of perlite addition on mechanical properties and wear resistance of alumina ceramics were investigated. Alumina powders including perlite 5\%,10\%,15\%,20\% and perlite free were prepared. These powders were shaped as rectangular by using uniaxial pressing system under the load of $100 \mathrm{MPa}$. The samples were sintered at $1400{ }^{\circ} \mathrm{C}-1550{ }^{\circ} \mathrm{C}$ for $2 \mathrm{~h}$ and were subjected to some mechanical tests such as hardness and fracture toughness. X-ray diffraction analysis was employed to determine the crystalline phases. Bulk densities of the samples were measured. In addition, erosive wear test was performed to investigate the erosive wear resistance of the samples. The wear test results were presented depending on different sliding speed and sintering temperature. These results showed that perlite addition has a positive effect on erosive wear resistance of the alumina ceramics, generally.
\end{abstract}

DOI: 10.12693 /APhysPolA.125.632

PACS: 81.05.Je, 81.05.Mh, 46.55.+d, 62.20.F-, 81.20.Ev

\section{Introduction}

Wear is one of the most encountered and important problem in engineering materials, leading to impairment of especially sliding components of mechanism. For instance: bearings, mechanical seals, high speed cutter sets, and medical prostheses. One of the industrial problems about wear process is erosive wear. The study of erosion of materials has become an important topic in the field of engineering materials. In fact, there has been quite an active research on both erosion testing and erosion mechanisms of metals, alloys, brittle materials during the last 30 years [1].

Erosive effect can affect the materials in the different mechanisms and conditions. The erodant particles can contact the fixed material or moving material can pull in the erosive media. Ductile materials will undergo wear by a process of plastic deformation in which the material is removed by the displacing or cutting action of the eroding particle. In a brittle material, on the other hand, the material will be removed from the formation and intersection of cracks that cause grain ejection from the surface of the tested material. Erosive wear involves several wear mechanisms which are largely controlled by the particle material, the angle of impingement, the impact velocity, and the particle size [2].

Alumina ceramics have found many applications and are commonly used engineering materials that can operate over a wide range of temperatures, loads, and different environmental conditions [3]. It has been widely used in seals and bearings due to low cost, a rigid structure, and a capability to retain smooth surfaces over a

*corresponding author; e-mail: sitas@itu.edu.tr broad range of contact parameters [4]. There is a lot of study about the improvement of the wear resistance of alumina ceramics in the literature $[5,6]$. In this study, the improvement possibilities of erosive wear resistance of alumina were investigated via perlite addition.

\section{Experimental procedure}

The raw materials were alumina powder (Alcoa, A16-SG) and perlite. The composition of perlite was given in Table. Perlite powders were added to alumina and mixed in different proportions (0-20 wt\%). To ensure a homogeneous mixture, each composition was milled in rubber-lined ceramic jars for $2 \mathrm{~h}$ using alumina balls and distilled water as the milling media and then sieved to pass through $38 \mu \mathrm{m}$. After drying in an incubator at $110{ }^{\circ} \mathrm{C}$ for $24 \mathrm{~h}$, the mixtures were granulated in moist conditions and then pressed at $100 \mathrm{MPa}$ to prepare rectangular shaped specimens with the size of $5 \times 8 \times 40 \mathrm{~mm}^{3}$.

TABLE I

The chemical composition of perlite.

\begin{tabular}{c|c}
\hline \hline $\mathrm{SiO}_{2}$ & 74.702 \\
$\mathrm{Al}_{2} \mathrm{O}_{3}$ & 14.966 \\
$\mathrm{MgO}$ & 0.762 \\
$\mathrm{Na}_{2} \mathrm{O}$ & 2.878 \\
$\mathrm{~K}_{2} \mathrm{O}$ & 4.652 \\
$\mathrm{CaO}$ & 0.852 \\
$\mathrm{Fe}_{2} \mathrm{O}_{3}$ & 0.788
\end{tabular}

All samples were sintered in an electric furnace with a heating rate of $5^{\circ} \mathrm{C} / \mathrm{min}$ at $1400,1450,1500$, and $1550{ }^{\circ} \mathrm{C}$ for $2 \mathrm{~h}$. After sintering, the bulk densities of sintered samples were measured. The crystalline phases of the samples were determined by X-ray diffraction (XRD) 
(Rigaku DMAX) with $\mathrm{Cu} K_{\alpha}(\lambda=1.54056 \AA)$ radiation. The microstructural characterization of fracture surfaces of sintered samples was examined using a JEOL JSM 6060 scanning electron microscopy (SEM). Furthermore, the hardness test was realized for mechanical properties observations. The mechanical properties were evaluated by Vickers microhardness indenter (HMV microhardness), load of 2000 gf for $10 \mathrm{~s}$; the measurements were carried out on five samples with five indentations.

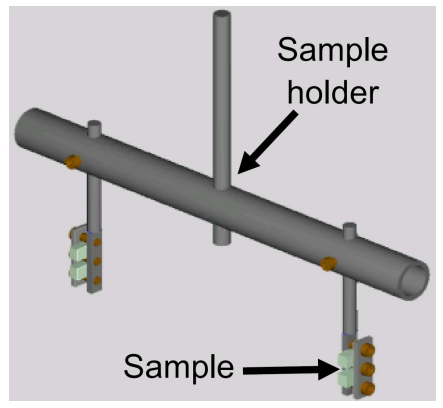

Fig. 1. Illustration of the erosive wear medium.

Erosion wear test was performed to investigate the erosive wear performance of the samples. Erosion wear test rig including a direct current motor and a vertical chuck with two arms was illustrated in Fig. 1. Sharp angular and brown colored corundum particle with a size of 36 mesh was preferred as erosive media. The samples were fixed into the sample holder. The container used for replacement of erodent materials was filled up with corundum. The erosion tests were performed to the perlite free alumina, and perlite reinforced alumina ceramics turning speed at 100, 150, and $200 \mathrm{rpm}$. Three tests were performed for each parameter for repeatability of the test results and average of the results was calculated.

In the erosive wear test, the samples were placed in the sample holder at the impact angles of $90^{\circ}$ and at $1000 \mathrm{~m}$ sliding distance. The samples were weighed to an accuracy of $0.001 \mathrm{mg}$ using an electronic balance before the wear test. After wear testing, erodent particles adhering to the surface of the sample were removed by air blasting. The weight loss of each sample was determined and normalized with surface area of the sample subjected to erosion test.

\section{Experimental results}

The XRD results showed that increase in sintering temperature provided better crystallization conditions. Crystalline peak intensities increased with increasing sintering temperature. This is related to better sintering in alumina based ceramics. The major phases detected by XRD were corundum (ASTM chart no. 98-000-0036), mullite (ASTM chart no. 01-073-1389) and sodium silicide (ASTM chart no. 01-074-0083).

Figure 2a indicates the hardness of the samples versus perlite addition. It was seen that perlite addition has a negative effect on the hardness. The hardness of the alumina base perlite reinforced ceramics depending on sintering temperature were measured to be $628-1260 \mathrm{Hv}$. Highest hardness value was obtained from perlite free samples. The hardness of the alumina is higher than perlite, because of this, increase in perlite content in the body provided decrease in hardness. On the other hand, increase in sintering temperature caused increase in hardness, markedly. Theoretical hardness of the sintered alumina is generally ranged from $1500 \mathrm{Hv}$ to $2100 \mathrm{Hv}$, according to different conditions such as sintering temperature. In this study, the hardness values of perlite free alumina samples are changing between 1390 and $2065 \mathrm{Hv}$ depending on sintering temperature.
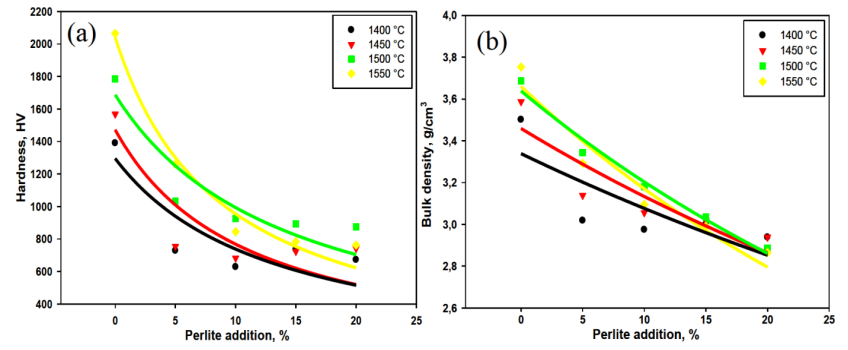

Fig. 2. The hardness results and bulk densities of the samples depending on perlite addition: (a) hardness, (b) bulk densities.

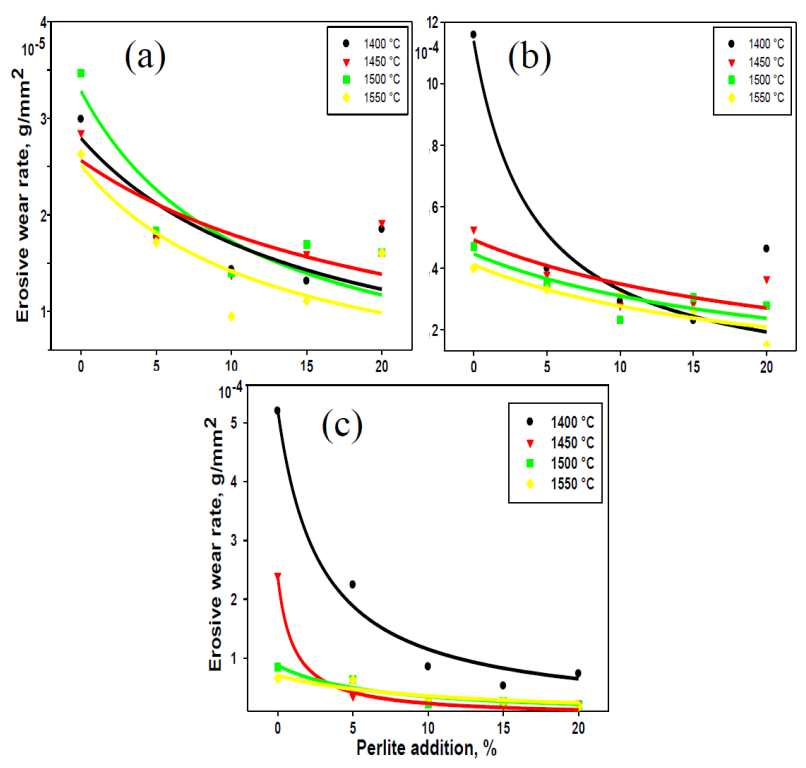

Fig. 3. Erosive wear test results in different impact velocities: (a) $100 \mathrm{rpm}$, (b) $150 \mathrm{rpm}$, (c) $200 \mathrm{rpm}$.

Figure $2 \mathrm{~b}$ shows bulk densities of the samples depending on perlite addition. These results exhibited good correlation with the hardness results. Highest bulk density to be $3.753 \mathrm{~g} / \mathrm{cm}^{3}$ was obtained from perlite free samples sintered at $1550^{\circ} \mathrm{C}$.

Erosive wear rate graphs of the samples versus perlite additions and impact velocity were given in Fig. 3 . 
Perlite has a positive effect on the erosion resistance of the samples. The other phases detected by XRD except for the corundum have lower hardness compared to alumina. It was possible that these phases deposit in the grain boundaries of alumina and they provided better toughness, more resistivity against repeated impacts. When the effect of sintering temperature on erosive wear was investigated, the erosion resistance increased with increase in sintering temperature. It is related to providing better sintering effect of high temperature. Impact velocity exhibited a negative effect on erosion. Erosive wear rates increased suddenly with increase in impact velocity. It was a predictable result, faster movement of erodent particles conducted more kinetic energy to the worn surface, possible. Erosive wear bases on the particles hitting the surface, repeatedly. To sum up, the shorter the amplitude of impact means the higher damage possibility on the surface.

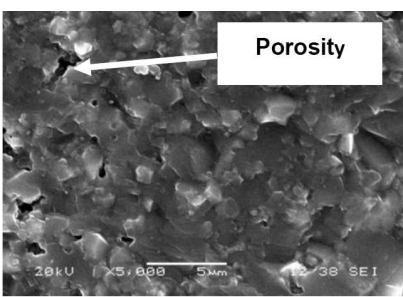

(a)

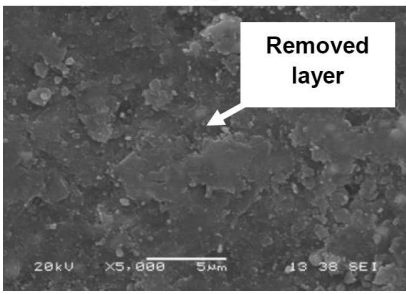

(c)

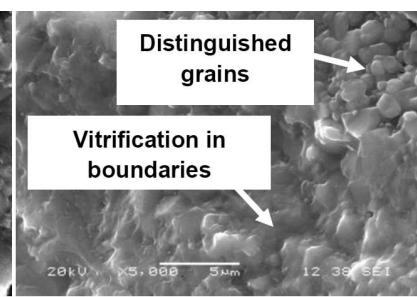

(b)

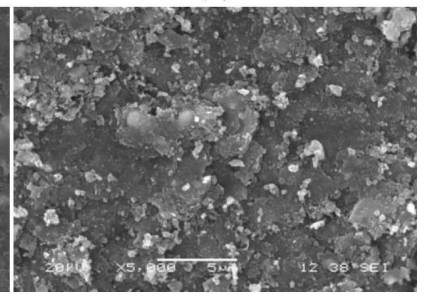

(d)
Fig. 4. SEM images obtained from cross-sectional and worn surface: (a) cross-sectional surface of the sample including 5\% perlite, (b) cross-sectional surface of the sample including $20 \%$ perlite, (c) worn surface of the sample including $5 \%$ perlite, (d) worn surface of the sample including $20 \%$ perlite.

SEM micrographs of the samples sintered at $1550^{\circ} \mathrm{C}$ for $2 \mathrm{~h}$ were given in Fig. 4. In Fig. $4 \mathrm{a}$,b, it can be seen that perlite addition provided increase in porosity. Also, it was confirmed by bulk density results. The differences between SEM image of the sample containing 5\% perlite and SEM image of the sample containing $20 \%$ perlite showed that it is difficult to distinguish the grain boundaries in the sample including higher perlite content. Perlite is a raw material containing high $\mathrm{SiO}_{2}$ content, because of this, vitrification and phase formation occurred in grain boundaries thanks to increase in perlite content, probably. SEM micrographs of worn surfaces exposed to an impact velocity of $100 \mathrm{rpm}$ are shown in Fig. 4c,d; repetitive erosive particle impacts caused the flake formation. In this damage mechanism, material loss occurs via flakes. Decreasing in hardness and ductile phase formation (sodium silicide) compared to corundum and mullite via perlite addition provided wear conditions exhibiting more toughness. Thus, harsh wear effects such as fracture and scraping have not been observed in this study.

\section{Summary}

In the current study, the effect of perlite addition on erosive wear performance of alumina base ceramics was investigated. Corundum, mullite, and sodium silicide phases were determined by XRD. Perlite addition caused decrease in hardness and bulk density. It was related to the differences of rheological density between perlite and alumina and differences of densities of crystallized phases. Perlite addition has a positive effect on the erosion resistance of the samples. It is possible that the surface including hard alumina grains and soft silica base phases in grain boundaries provided sufficient conditions for erosion tests. The microstructural analysis of the worn surface showed that the damage mechanism was dominated by flake formation and removed layer after the erosion test.

\section{References}

[1] Q. Fang, P. Sidky, M.G. Hocking, Mater. Des. 18, 389 (1997).

[2] E. Ercenk, U. Sen, S. Yilmaz, Tribol. Int. 52, 94 (2012).

[3] M. Kalin, S. Novak, J. Vižintin, Wear 254, 1141 (2003).

[4] Y. Wang, S.M. Hsu, Wear 195, 90 (1996).

[5] Y. Morita, K. Nakata, K. Ikeuchi, Wear 254, 147 (2003).

[6] Y. Wang, Y. Yang, Y. Zhao, W. Tian, H. Bian, J. He, Wear 266, 1051 (2009). 